\title{
Amiloidosis cardíaca: revisión de una serie de casos
}

\author{
Cardiac amyloidosis: a review of a series of cases \\ Marina Povar-Echeverría1*, Pablo E. Auquilla-Clavijo², Bienvenido J. Povar-Marco³, \\ Eva M. Moreno-Esteban ${ }^{2}$ y María P. Figueras-Villalba ${ }^{1}$ \\ ${ }^{1}$ Servicio de Medicina Interna; ${ }^{2}$ Servicio de Cardiología; ${ }^{3}$ Servicio de Urgencias. Hospital Universitario Miguel Servet, Zaragoza, España
}

\begin{abstract}
Resumen
Antecedentes y objetivo: La amiloidosis cardíaca es una entidad que permanece infradiagnosticada, a pesar de los avances recientes en su diagnóstico y tratamiento. El objetivo de este estudio es revisar una serie de casos de amiloidosis cardíaca para describir los principales datos clínicos y los hallazgos en las pruebas de imagen. Materiales y métodos: Estudio retrospectivo de pacientes con diagnóstico principal o secundario de amiloidosis cardíaca en los informes de alta de pacientes hospitalizados en este centro desde 2006 hasta 2016. Se revisaron los datos clínicos de los pacientes, así como las pruebas de imagen (ECG, ecocardiograma, gammagrafía cardíaca, resonancia magnética cardíaca). Se realizó seguimiento de los pacientes hasta enero de 2018. Resultados: Se analiza a 30 pacientes (20 varones) con media de 65 años. Los principales datos ecocardiográficos fueron dilatación biauricular, disfunción diastólica e hipertrofia ventricular izquierda (HVI) en un 97\%. Sólo el 6.7\% cumplía criterios de HVI en el electrocardiograma. Hasta un 33\% tenía disfunción sistólica. Se realizó gammagrafía y resonancia magnética cardíaca en un 33\%. La supervivencia a los 12 meses fue de 61\%. Conclusión: La presencia de insuficiencia cardíaca, fibrilación auricular o trastornos de conducción junto a datos ecocardiográficos indicativos debe alertar al clínico. Otros datos como disfunción sistólica o sexo femenino no deben disminuir la sospecha. El estudio debe completarse con gammagrafía y resonancia magnética cardíaca, ya que el diagnóstico temprano tiene implicaciones pronósticas y terapéuticas.
\end{abstract}

Palabras clave: Amiloidosis. Transtirretina. Miocardiopatía. Insuficiencia cardíaca. ${ }^{99 m} T c-D P D$ gammagrafía.

\begin{abstract}
Background and objective: Cardiac amyloidosis is an entity that remains underdiagnostic, despite recent advances in its diagnosis and treatment. The aim of this study is to review a series of diagnosed cases of cardiac amyloidosis to describe the main clinical data and the findings in the imaging tests. Materials and methods: Retrospective study of patients with primary or secondary diagnosis of cardiac amyloidosis in discharge reports of patients hospitalized in our center from 2006 to 2016. The clinical data of the patients were reviewed, as well as the imaging tests (ECG, echocardiogram, cardiac scintigraphy, cardiac magnetic resonance). Patients were followed until January 2018. Results: We analyze 30 patients (20 men) with an average of 65 years. The main echocardiographic data were biatrial dilatation, diastolic dysfunction and left ventricular hypertrophy (LVH) in 97\%. Only 6.7\% met criteria for LVH in the electrocardiogram. Up to 33\% had systolic dysfunction.
\end{abstract}

\section{Correspondencia:}

*Marina Povar-Echeverría

E-mail: marinapovar89@gmail.com
Disponible en internet: 23-01-2020 Arch Cardiol Mex. 2020;90(3):259-265 www.archivoscardiologia.com 1405-9940 @ 2019 Instituto Nacional de Cardiología Ignacio Chávez. Publicado por Permanyer. Este es un artículo open access bajo la licencia CC BY-NC-ND (http://creativecommons.org/licenses/by-nc-nd/4.0/). 
Scintigraphy and cardiac magnetic resonance were performed in 33\%. Survival at 12 months was $61 \%$. Conclusion: The presence of heart failure, atrial fibrillation or conduction disorders with suggestive echocardiographic data should alert the clinician. Other data such as systolic dysfunction or female sex should not decrease the suspicion. The study should be completed with gammagraphy and cardiac magnetic resonance since early diagnosis has prognostic and therapeutic implications.

Key words: Amyloidosis. Transthyretin. Cardiomiopathy. Heart failure. ${ }^{99 m} T c-D P D$ scintigraphy.

\section{Introducción}

La amiloidosis cardíaca se produce por el depósito de amiloide en los miocitos, que ocasiona una miocardiopatía con fisiopatología restrictiva. Aunque existen más de 30 proteínas conocidas que pueden producir depósito de amiloide, sólo cinco lo hacen de forma significativa en el plano cardiaco: cadenas ligeras, que causan amiloidosis primaria (AL), transtirretina (TTR), apolipoproteína $A(A A p o A-1)$, fibrinógeno y componente $A$ sérico, que da lugar a amiloidosis secundaria (AA). En la actualidad se considera que la amiloidosis cardíaca por transtirretina (ATTR) podría ser más prevalente que la $A L^{1}$, por lo regular considerada la más frecuente. Existe una ATTR hereditaria, con más de 100 mutaciones descritas, y una ATTR adquirida que se conoce como wild type (ATTRwt).

La ATTRwt es una entidad descrita de forma predominante en varones entre la sexta y la séptima década. Su prevalencia no se conoce con exactitud, pero es probable que sea una entidad infradiagnosticada ${ }^{1}$. Estudios realizados en necropsias muestran que la prevalencia de depósito de TTR en personas mayores de 80 años alcanza el $25 \%^{2}$. En necropsias de pacientes con insuficiencia cardíaca y fracción de expulsión preservada (ICFEp) se demostró depósito moderado a grave en un $5 \%{ }^{3}$. Otros estudios realizados mediante pruebas no invasivas han determinado que la prevalencia de ATTR en pacientes con ICFEp e hipertrofia ventricular izquierda $(\mathrm{HVI})$ podría ser del 13\%4,5. Estos datos sugieren que la ATTRwt podría ser una de las principales causas de insuficiencia cardíaca con fracción de expulsión preservada.

En los últimos años se han producido avances importantes en el diagnóstico y tratamiento de la ATTR. Los criterios diagnósticos que publicaron Gillmore, et al. ${ }^{6}$ permiten establecer el diagnóstico de la enfermedad de forma no invasiva y quizá en estadios más tempranos. Además, el estudio ATTR-ACT ha demostrado que el tafamidis, un fármaco que estabiliza a la transtirretina al evitar la disociación del tetrámero y su depósito en los tejidos, disminuye la mortalidad por todas las causas y por origen cardiovascular, y retrasa el empeoramiento de la capacidad funcional y la calidad de vida ${ }^{5}$. Por todo ello se considera de interés realizar este estudio con el objetivo de describir las características clínicas y los hallazgos de las pruebas diagnósticas de imagen de una cohorte de pacientes con amiloidosis cardíaca diagnosticados en el centro de los autores.

\section{Material y métodos}

Estudio descriptivo y retrospectivo de los pacientes diagnosticados de amiloidosis cardíaca en un hospital de tercer nivel desde el 1 de enero de 2006 hasta el 31 de septiembre de 2016. Se seleccionaron los informes de alta con diagnóstico principal o secundario de amiloidosis (código 277.3 de CIE-9). Se excluyeron los episodios repetidos de un mismo paciente, a los individuos con diagnóstico de angiopatía amiloide cuya clínica era exclusivamente neurológica y a los enfermos con amiloidosis sistémica sin datos de afectación cardiológica clínica ni por técnicas de imagen. El proceso de selección de los pacientes se muestra en la figura 1.

Se revisó la documentación clínica de cada uno de los pacientes y se registraron los siguientes datos: edad, sexo, antecedentes personales (factores de riesgo cardiovascular, antecedentes familiares de amiloidosis e ingresos previos), clínica de presentación (síntomas cardíacos y extracardíacos), pruebas diagnósticas realizadas y supervivencia. La presencia de voltajes bajos en el electrocardiograma (ECG) se identificó por una amplitud de QRS $<1 \mathrm{mV}$ en todas las derivaciones precordiales $0<0.5 \mathrm{mV}$ en las de los miembros. Para definir la hipertrofia ventricular en el ECG se empleó el criterio de Sokolow (suma de onda $\mathrm{S}$ en V1 y onda R en V5 o V6 > $3.5 \mathrm{mV}$ ). El patrón de seudoinfarto se definió como alteraciones en el ECG que simularan un infarto de miocardio en ausencia de éste. Se consideró angina microvascular a la clínica de angor con coronariografía sin lesiones significativas. La biopsia era concluyente si demostraba depósito de material amiloide. El seguimiento de los pacientes se prolongó hasta el 31 de enero de 2018, con un periodo 


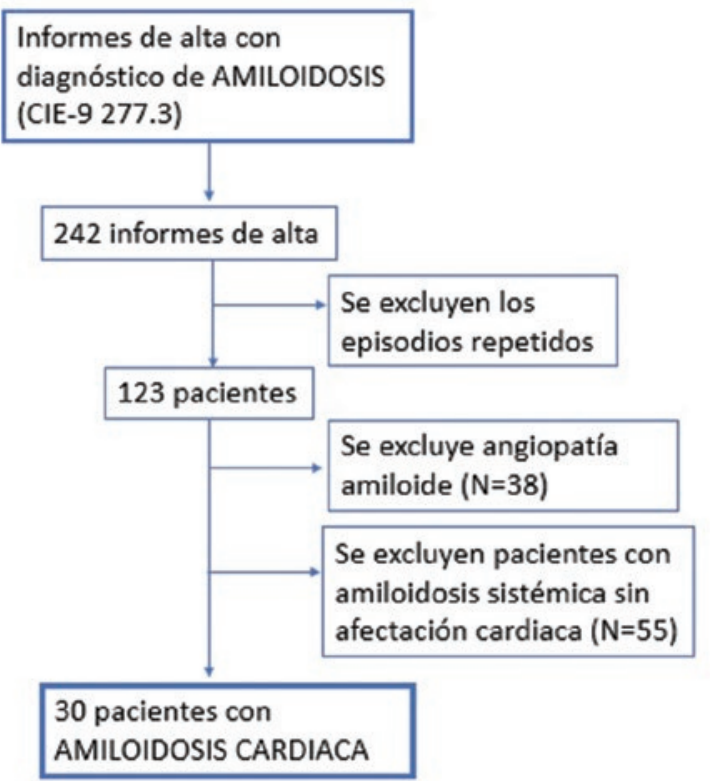

Figura 1. Algoritmo que demuestra el proceso de selección de los pacientes.

mínimo de seguimiento de 16 meses, y se efectuó al analizar la documentación disponible en la historia clínica electrónica y mediante contacto telefónico cuando fue necesario.

El análisis estadístico se realizó con el programa IBM SPSS Statistics 19. Las variables cuantitativas eran paramétricas, mediante la prueba de Shapiro-Wilk, por lo que se presentan como media y desviación estándar; las variables cualitativas se presentan como frecuencia y porcentaje (\%). La supervivencia se evaluó mediante la curva de Kaplan-Meier y la prueba de logrank. El Comité Ético de Investigación Clínica de Aragón autorizó el estudio.

\section{Resultados}

Se incluyó a 30 pacientes, 20 varones y 10 mujeres, con una edad media de 65 años. La amiloidosis más frecuente fue la primaria $(A L)$ presente en 10 pacientes (33\%), en cinco era hereditaria (4 ATTR y 1 AApoA-1), en dos secundaria (AA) y en dos ATTRwt. En 11 sujetos $(36,7 \%)$ no se identificó el tipo de amiloidosis.

En el momento del diagnóstico, casi un $60 \%$ de los pacientes tenía ingresos previos por insuficiencia cardíaca, un $20 \%$ era portador de marcapasos y un $10 \%$ se había sometido a intervención por síndrome de túnel carpiano. La presentación clínica predominante fue la insuficiencia cardíaca (90\%), seguida de los trastornos de conducción que precisaron implante de marcapasos (40\%); el $43 \%$ de los pacientes presentaba fibrilación auricular (FA) y el $13 \%$ angina microvascular. Los síntomas clínicos de presentación, cardíacos y extracardíacos se exponen en la tabla 1.

En relación con los hallazgos en el ECG, un 50\% tenía algún tipo de trastorno de conducción y también fueron frecuentes los patrones electrocardiográficos de bajos voltajes y el de seudoinfarto; sólo un $6.7 \%$ de los pacientes satisfacía los criterios de HVI. El $93 \%$ de los individuos cursaba con cardiomegalia en la radiografía de tórax. En el ecocardiograma, el 96\% mostraba hipertrofia ventricular izquierda y el $33 \%$ cursaba con fracción de expulsión de ventrículo izquierdo (FEVI) deprimida; el $86 \%$ revelaba algún grado de alteración de la función diastólica, las más de las veces de grado III (patrón restrictivo). Sólo un 26\% mostró el patrón granular o sparkling miocárdico. El $80 \%$ tenía algún grado de dilatación auricular izquierda y un $53 \%$ de aurícula derecha. El 30\% acusaba dilatación y disfunción de ventrículo derecho. En cuanto a las valvulopatías se evidenció un $20 \%$ de pacientes con algún grado de estenosis aórtica. El resto de los parámetros ecocardiográficos se detalla en la tabla 1. Se realizó resonancia magnética cardíaca en 10 pacientes, que fue diagnóstica en todos los casos: en siete de los sujetos se observó realce tardío de gadolinio subendocárdico global y en tres distribución en parches. Sólo en cuatro de los nueve pacientes, la gammagrafía cardíaca demostró captación miocárdica. En 27 individuos (90\%) se efectuó estudio anatomopatológico. Las muestras se tomaron mediante aspirado de grasa abdominal en seis casos $(20 \%)$ o biopsia renal en seis $(20 \%)$, de mucosa rectal en tres $(10 \%)$, endomiocárdica en tres $(10 \%)$, glándula salivar en uno $(3 \%)$, entre otros. El estudio sólo fue concluyente en 20 de ellos.

Durante una mediana de seguimiento de 16 meses (IC95\%, 16-72) desde el diagnóstico, 21 pacientes fallecieron (un 70\% del total). La mediana de supervivencia fue de 21 meses, con una edad media de 69 años en el momento del deceso. La supervivencia a los 12 , 24 y 36 meses fue de $61.6 \%$ (IC 95\%, 41-76), 46\% (IC $95 \%, 27-63)$ y $42 \%$ (IC 95\%, 24-60), respectivamente (Fig. 2).

\section{Discusión}

Este estudio describe una cohorte de pacientes diagnosticados con amiloidosis cardíaca que posee características generales similares a las encontradas en 
Arch Cardiol Mex. 2020;90(3)

Tabla 1. Características clínicas, electrocardiográficas y parámetros ecocardiográficos de los pacientes

Presentación clínica

Clínica cardiovascular
Total $(\mathrm{n}=30)$

Pruebas complementarias

Ecocardiografía transtorácica

\begin{tabular}{|c|c|c|c|}
\hline \multicolumn{2}{|c|}{ Clínica cardiovascular } & \multicolumn{2}{|c|}{ Ecocardiografía transtorácica } \\
\hline Insuficiencia cardíaca & 28 (93.3) & Hipertrofia VI & $29(96.7)$ \\
\hline Angina & $7(23.3)$ & Grosor tabique interventricular* & $16.8 \pm 3.3$ \\
\hline Enfermedad coronaria arterial & $3(10)$ & Función sistólica VI preservada & $20(66.7)$ \\
\hline Angina microvascular & $4(13,3)$ & Disfunción sistólica VI & $10(33.3)$ \\
\hline Síncope & $8(26.7)$ & Leve & $6(20)$ \\
\hline Implante de marcapasos & $12(40)$ & Moderada & $4(13.3)$ \\
\hline Bloqueo auriculoventricular & $7(23.3)$ & Grave & 0 \\
\hline Síndrome bradi-taqui & $2(6.7)$ & $\mathrm{FEVI}{ }^{*}$ & $54 \pm 12.6$ \\
\hline Ambas & $3(10)$ & Patrón granular miocárdico & $8(26.7)$ \\
\hline Arritmias supraventriculares & $13(43.3)$ & Disfunción diastólica & $26(86.7)$ \\
\hline Arritmias ventriculares & $6(20)$ & Disminución E/a & $8(26.7)$ \\
\hline Pericarditis & $1(3.3)$ & Patrón seudonormal & $4(13.3)$ \\
\hline Tromboembolismo periférico & $2(6.7)$ & Patrón de Ilenado restrictivo & $14(46,7)$ \\
\hline \multicolumn{2}{|c|}{ Síntomas extracardíacos } & Dilatación Al & $24(80)$ \\
\hline Neuropatía & $6(20)$ & Dilatación AD & $16(53.3)$ \\
\hline Hepatomegalia & $13(43.3)$ & Dilatación VD & $9(30)$ \\
\hline Anemia & $17(56.7)$ & Disfunción VD & $9(30)$ \\
\hline Insuficiencia renal & $16(53.3)$ & TAPSE* & $16.4 \pm 2.7$ \\
\hline Síndrome nefrótico & $9(30)$ & PAPS* & $43 \pm 10$ \\
\hline Enfermedad hematológica & $8(26.7)$ & Estenosis aórtica & $6(20)$ \\
\hline Linfoma & $1(3.3)$ & Ligera & $4(13.3)$ \\
\hline Mieloma múltiple & $5(16.7)$ & Moderada & $2(6.7)$ \\
\hline GMSI & $2(6.7)$ & Insuficiencia aórtica & $7(23.3)$ \\
\hline \multicolumn{2}{|c|}{ Pruebas complementarias } & Ligera & $4(13.3)$ \\
\hline Electrocardiograma & & Moderada & $3(10)$ \\
\hline Voltajes bajos & $8(26.7)$ & Insuficiencia mitral & $20(66.7)$ \\
\hline Criterios de hipertrofia de VI & $2(6.7)$ & Ligera & 14 (46.7) \\
\hline Patrón de seudoinfarto & $8(26.7)$ & Moderada & $6(20)$ \\
\hline Trastorno de conducción & $15(50)$ & Trombo intracardíaco & $1(3.3)$ \\
\hline BAV primer grado & $1(3,3)$ & Derrame pericárdico & $10(33.3)$ \\
\hline BAV segundo grado & $1(3,3)$ & Ligero & $5(16.7)$ \\
\hline BAV avanzado & $4(13,3)$ & Moderado & $1(3.3)$ \\
\hline Trastorno intraventricular & $9(30)$ & Grave & $4(13.3)$ \\
\hline
\end{tabular}

Total $(n=30)$

* Media y desviación estándar.

VI: ventrículo izquierdo; BAV: bloqueo auriculoventricular; GMSI: gammapatía monoclonal de significado incierto; FEVl: fracción de expulsión de ventrículo izquierdo; AI: aurícula izquierda; AD: aurícula derecha; VD: ventrículo; PAPS: presión arterial pulmonar sistólica; TAPSE: desplazamiento sistólico del anillo tricuspídeo derecho. 


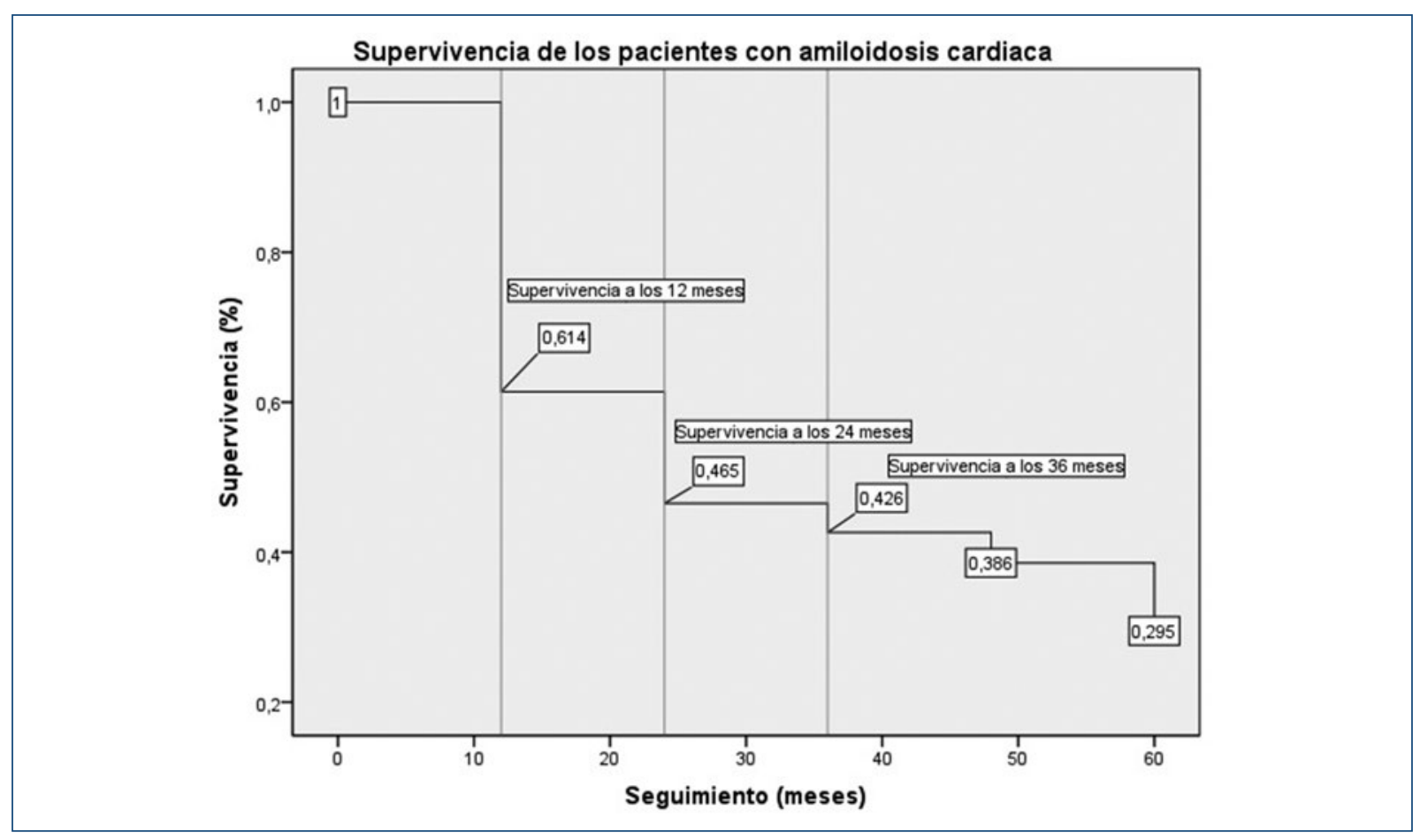

Figura 2. Supervivencia global de los pacientes con amiloidosis cardíaca.

estudios previos, con un predominio de pacientes de sexo masculino y edad avanzada y con insuficiencia cardíaca como forma más frecuente de presentación. Los datos obtenidos permiten emitir algunas consideraciones sobre el proceso diagnóstico de esta enfermedad, dada la creciente accesibilidad de las pruebas diagnósticas de imagen cardíaca y los nuevos criterios propuestos para el diagnóstico no invasivo ${ }^{6}$.

La insuficiencia cardíaca fue la forma de presentación más frecuente en esta serie, seguida por los trastornos de conducción con necesidad de implante de marcapasos, en este caso con porcentajes más elevados que los descritos en estudios previos en los que sólo un $7 \%$ de casos tuvo esta presentación clínica ${ }^{7}$. Aunque un porcentaje considerable de los pacientes tenía antecedentes de insuficiencia cardíaca o implante de marcapasos, la enfermedad había pasado inadvertida, incluso en presencia de datos típicos como el síndrome del túnel carpiano o síntomas extracardíacos. Es conocida la importancia de mantener un elevado índice de sospecha para establecer un diagnóstico temprano ${ }^{8}$, lo que evita oportunidades perdidas de diagnóstico. En este sentido, a pesar del habitual predominio masculino de esta enfermedad, el sexo femenino no debe reducir el umbral de sospecha ya que un tercio de los pacientes correspondía a mujeres, un porcentaje incluso superior al 18 a $20 \%$ de otros estudios ${ }^{7}$.

Otros datos que deben tomarse en consideración son la HVI en ausencia de causas evidentes, como hipertensión arterial no controlada o valvulopatías, o la coexistencia de bajos voltajes en el ECG y de HVI en el ecocardiograma ${ }^{1,7,9}$. En esta serie, el $96 \%$ de los casos tenía HVI frente a un $6.7 \%$ que cumplía el criterio de HVI en el ECG, como expresión del depósito de amiloide y no de la hipertrofia de miocitos. El valor medio para el tabique interventricular en estos pacientes fue de $16 \mathrm{~mm}$, superior al descrito en series anteriores $^{1}$, lo que sugiere que probablemente la mayoría de ellos se hallaba en fases avanzadas de la enfermedad.

Si bien la mayoría de los pacientes muestra ICFEp, la sospecha de amiloidosis no debe restringirse a este grupo, puesto que la función contráctil empeora a medida que el depósito de amiloide progresa, y pueden existir distintos grados de disfunción sistólica, como lo reveló un $33 \%$ de los pacientes de este estudio ${ }^{1}$. Estos datos confirman los hallazgos de estudios previos, en los que los pacientes con $\mathrm{FEVI}<50 \%$ alcanzan porcentajes de 37 a $50 \%$.1,10. Debe tenerse en cuenta que la valoración de la función sistólica en la amiloidosis mediante la técnica estándar (FEVI por el método de Simpson biplanar) 
tiene limitaciones, dado que valores ligeramente deprimidos implican ya un daño miocárdico relevante 1 . Algunas modalidades ecocardiográficas novedosas como el strain por speckle-tracking proporcionan índices más adecuados de función sistólica y permiten la detección de la afectación miocárdica en fases más tempranas ${ }^{11}$. El strain global longitudinal del ventrículo izquierdo suele estar significativamente reducido en esta entidad en particular a expensas de los segmentos basales y medios con valores preservados en los segmentos apicales (patrón típico de "cereza apical")12. Valores reducidos del strain global longitudinal se han relacionado de forma independiente con peor pronóstico en el seguimiento de pacientes con amiloidosis cardíaca ${ }^{13}$.

Otro de los hallazgos ecocardiográficos frecuente en esta serie fue la dilatación biauricular, sobre todo de aurícula izquierda, que se correspondía con porcentajes similares de disfunción diastólica. Éstos son hallazgos típicos de esta enfermedad y están descritos con porcentajes similares en trabajos previos ${ }^{2}$. El patrón granular del miocardio o sparkling miocárdico, considerado un hallazgo típico, sólo estaba presente en el $26 \%$ de los pacientes y se ha encontrado también en bajos porcentajes en otras series ${ }^{9}$. Por lo tanto, la sospecha diagnóstica de amiloidosis cardíaca no debe apoyarse en este dato aislado, sino en el conjunto de los signos ecocardiográficos descritos con anterioridad.

La restricción progresiva al llenado ventricular con aumento de las presiones telediastólicas y dilatación secundaria de aurícula izquierda favorece una alta prevalencia de FA, observada en series previas ${ }^{7}$ y que alcanza el $43 \%$ en este estudio. El grupo de la Clínica Mayo ha planteado que debe descartarse ATTRwt ante el diagnóstico de FA no valvular en ancianos ${ }^{10}$. También destaca la prevalencia de un $20 \%$ de estenosis aórtica en esta serie, que se corresponde con los hallazgos de varios estudios recientes que muestran la presencia de ATTRwt hasta en $6.5 \%$ de los pacientes sometidos a recambio de válvula aortica ${ }^{14}$. En este estudio se evidenció un peor pronóstico postoperatorio en este subgrupo de pacientes ${ }^{14}$. Estos datos llevan a considerar si sería necesario descartar la presencia de ATTRwt en individuos de edad avanzada con estenosis aórtica grave antes de plantear la conducta terapéutica.

Este trabajo es anterior a la publicación de los criterios de diagnóstico no invasivo para ATTR propuestos en $2016^{6}$, por lo que la gammagrafía y la RMN se utilizaron poco y la biopsia se realizó en prácticamente todos los casos, con escasa rentabilidad: en un $33 \%$ de los casos no fue concluyente y sólo se realizó la biopsia endomiocárdica, técnica de referencia en tres pacientes. La rentabilidad diagnóstica de la biopsia extracardíaca en amiloidosis con afectación exclusivamente cardíaca es poco conocida ${ }^{1}$, pero se ha descrito que la sensibilidad de la aspiración de la grasa abdominal es menor al $15 \%^{15}$ y no debe olvidarse que la biopsia negativa de un órgano no afectado no excluye el diagnóstico. En la actualidad, los criterios no invasivos $^{6}$ permiten el diagnóstico de ATTRwt con una sensibilidad y un valor predictivo positivo del $100 \%$ sin necesidad de confirmación histológica. Estos criterios son insuficiencia cardíaca con prueba de imagen (ecocardiografía o resonancia magnética cardíaca) indicativa de amiloidosis, gammagrafía con captación cardíaca (grados $2 \circ 3$ ) de radiotrazador ${ }^{99 \mathrm{~m} T c-D P D,}{ }^{99 \mathrm{~m} T c-P Y P}$ o ${ }^{99 m} T c-H M D P$ y ausencia de proteína monoclonal detectable mediante inmunofijación en sangre y orina ${ }^{6,16}$. Es probable que la gammagrafía haya establecido el diagnóstico de ATTRwt en alguno de los pacientes en los que la biopsia no fue concluyente, por lo que debe implementarse de forma generalizada en pacientes con perfiles clínicos consistentes con este diagnóstico.

La supervivencia de los pacientes, incluidos todos los tipos de amiloidosis, fue del $61 \%$ a los 12 meses, notablemente inferior al 93\% que publicó González-López ${ }^{7}$ en una serie de casos de ATTRwt. El pronóstico de la ATTRwt es mejor que los otros tipos, con una mediana de supervivencia de 75 meses respecto de 13 en la primaria ${ }^{9}$. Para determinar el pronóstico de la ATTRwt se ha publicado en fecha reciente una clasificación de estadificación basada en el filtrado glomerular y el pro-BNP ${ }^{16}$.

La principal limitación de este trabajo es que se basa en una revisión retrospectiva de las historias clínicas, lo que implica un posible sesgo en la obtención de la información. El estudio describe las características clínicas de los pacientes con amiloidosis cardíaca en un centro único y en consecuencia los hallazgos pueden no ser generalizables y, además, el tamaño de la muestra es reducido. Dado que esta entidad está probablemente infradiagnosticada, este estudio no ha tenido en cuenta las características clínicas y los hallazgos ecocardiográficos de este grupo de pacientes. Sería de gran interés realizar un estudio prospectivo multicéntrico basado en los criterios de diagnóstico no invasivo para establecer la prevalencia real de la ATTRwt y sus características clínicas.

\section{Conclusión}

La presencia de insuficiencia cardíaca, fibrilación auricular, trastornos de conducción e incluso 
estenosis aórtica, junto con signos ecocardiográficos como hipertrofia ventricular, dilatación biauricular y disfunción diastólica avanzada, debe alertar sobre la presencia de esta entidad. Otros datos como disfunción sistólica o sexo femenino no deben atenuar la sospecha clínica. El estudio debe completarse con gammagrafía y resonancia magnética cardíaca, ya que el diagnóstico temprano tiene implicaciones pronósticas y terapéuticas.

\section{Financiamiento}

La presente investigación no ha recibido ayuda específica proveniente de agencias del sector público, sector comercial o entidades sin ánimo de lucro.

\section{Conflicto de intereses}

Ninguno.

\section{Responsabilidades éticas}

Protección de personas y animales. Los autores declaran que para esta investigación no se han realizado experimentos en seres humanos ni en animales.

Confidencialidad de los datos. Los autores declaran que han seguido los protocolos de su centro de trabajo sobre la publicación de datos de pacientes.

Derecho a la privacidad y consentimiento informado. Los autores declaran que en este artículo no aparecen datos de pacientes.

\section{Bibliografía}

1. González López E, López Sainz A, García Pavía P. Diagnóstico y tratamiento de la amiloidosis cardíaca por transtirretina. Progreso y esperanza. Rev Esp Cardiol. 2017;70(11):999-1004.

2. Maurer MS, Elliott $P$, Comenzo R, Semigran M, Rapezzi C. Addressing common questions encountered in the diagnosis and management of cardiac amyloidosis. Circulation 2017;135:1357-1377.

3. Mohammed SF, Mirzoyev SA, Edwards WD, Dogan A, Grogan DR, Dunlay SM, et al. Left ventricular amyloid deposition in patients with heart failure and preserved ejection fraction. JACC Heart Fail. 2014;2(2):113-122.

4. González-López E, Gallego-Delgado M, Guzzo-Merello G, de Haro-Del Moral FJ, Cobo-Marcos M, Robles C, et al. Wild-type transthyretin amyloidosis as a cause of heart failure with preserved ejection fraction. Eur Heart J. 20157;36(38):2585-94.

5. Maurer MS, Schwartz JH, Gundapaneni B, Elliott PM, Merlini G, Waddington-Cruz $\mathrm{M}$, et al. ATTR-ACT Study Investigators. tafamidis treatment for patients with transthyretin amyloid cardiomyopathy. $\mathrm{N}$ Engl $\mathrm{J}$ Med. 2018;379(11):1007-1016.

6. Gillmore JD, Maurer MS, Falk RH, Merlini G, Damy T, Dispenzieri A et al. Nonbiopsy diagnosis of cardiac transthyretin amyloidosis. Circulation 2016;133(24):2404-12.

7. González-López E, Gagliardi C, Domínguez F, Quarta CC, de Haro-De Moral FJ, Milandri A, et al. Clinical characteristics of wildtype transthyretin cardiac amyloidosis: disproving myths. Eur Heart J. 2017;38(24):1895-1904.

8. Culotta V, Moon JC. Amiloidosis cardíaca por transtirretina: antes una enfermedad monogénica minoritaria, ¿ahora una vía común de insuficiencia cardíaca? Rev Esp Cardiol. 2016;69:888-889.

9. García-Pavía P, Tomé-Esteban MT, Rapezzi C. Amiloidosis. También una enfermedad del corazón. Rev Esp Cardiol. 2011;64:797-808.

10. Grogan M, Scott CG, Kyle RA, Zeldenrust SR, Gertz MA, Lin G, et al. Natural history of wild-type transthyretin cardiac amyloidosis and risk stratification using a novel staging system. J Am Coll Cardiol. 2016;68:1014-1020.

11. Gertz MA, Benson MD, Dyck PJ, Grogan M, Coelho T, Cruz M, et al. Diagnosis, prognosis, and therapy of transthyretin amyloidosis. J Am Coll Cardiol. 2015;66:2451-2466.

12. Tops LF, Delgado V, Marsan NA, Bax JJ. Myocardial strain to detect subtle left ventricular systolic dysfunction. Eur J Heart Fail. 2017;19(3):307-313.

13. Buss SJ, Emami M, Mereles D, Korosoglou G, Kristen AV, Voss A, et al. Longitudinal left ventricular function for prediction of survival in systemic light-chain amyloidosis: incremental value compared with clinical and biochemical markers. J Am Coll Cardiol. 2012;60(12):1067-76.

14. Treibel TA, Fontana M, Gilbertson JA, Castelletti S, White SK, Scully PR, et al. Occult transthyretin cardiac amyloid in severe calcific aortic stenosis: prevalence and prognosis in patients undergoing surgical aortic valve replacement. Circ Cardiovasc Imaging 2016;9(8). pii: e005066.

15. Quarta CC, González-López E, Gilbertson JA, Botcher N, Rowczenio D, Petrie A, et al. Diagnostic sensivity of abdominal fat aspiration in cardiac amyloidosis. EHJ 2017;38:1905-1908.

16. Gillmore JD, Damy T, Fontana M, Hutchinson M, Lachmann HJ, Martínez-Naharro A, et al. A new staging system for cardiac transthyretin amyloidosis. Eur Heart J 2017. doi: 10.1093/eurheartj/ehx589. 